\title{
Kyste de la vésicule séminale associé à une agénésie rénale homolatérale : à propos de trois observations
}

\author{
Ahmed CHOUKET*, Mounir LEFI, Abdelkader BOUHLEL, Abdelahad OUNAIES, Hamadi SAAD* \\ Service d'Urologie - Hôpital les Aghlabites, Kairouan, Tunisie ; \\ * Service d'urologie et Transplantation rénale, CHU Fattouma Bourguiba- Monastir, Tunisie
}

\section{RESUME}

L'association d'un kyste de la vésicule séminale (KVS) à une agénésie rénale ipsilatérale est une anomalie congénitale rare. Cependant cette pathologie est de plus en plus diagnostiquée, grâce au progrès de l'imagerie ces dernières années.

Les auteurs rapportent deux observations d'un KVS associé à une agénésie rénale homolatérale et une troisième de même type, associée à une ectopie urétérale. Ils rappellent l'étiopathogénie de cette anomalie séminale et sa relation avec la dysgénésie rénale, et ils revoient les moyens diagnostiques et thérapeutiques possibles dans cette affection.

Mots clés : vésicule séminale, kyste, uretère ectopique, agénésie rénale, kystectomie, incision endoscopique

\section{INTRODUCTION}

Le kyste de la vésicule séminale associé à une malformation rénale est une entité rare. Elle touche moins de $0,005 \%$ de la population générale [2].

En 1914, Zinner, décrivait pour la première fois une telle association d'un kyste congénital de la vésicule séminale et d'une agénésie rénale ipsilatérale [20]. Mais le premier cas de cette affection associée à un uretère ectopique abouché dans le kyste a été rapporté par Zaontz et Kass [19].

L'obstruction congénitale de la zone de la jonction entre la vésicule séminale et le canal éjaculateur est considérée comme le facteur causal le plus probable. La relation entre l'existence d'un KVS et d'autres anomalies de la voie urinaire est bien connue, en particulier l'association d'une ectopie urétérale et d'une hypoplasie ou d'une agénésie rénale a été décrite plusieurs fois [11].

\section{CAS CLINIQUES}

\section{Observation $\mathrm{N}^{\circ} \mathbf{1}$}

K.A., âgé de 20 ans, sans antécédents particuliers, est hospitalisé au service d'urologie pour exploration d'une dysurie, pollakiurie et parfois des brûlures mictionnelles, évoluant depuis plusieurs mois. L'examen physique et la fonction rénale étaient normaux et l'examen cytobactériologique des urines était stérile.

L'échographie pelvienne avait montré la présence d'une masse kystique de $3,5 \mathrm{~cm}$ de grand axe, postérieure par rapport à la vessie et faisant sailli intravésicale (Figure 1). L'urographie intraveineuse avait objectivé une image lacunaire droite sur le cystogramme avec un rein droit muet (Figure 2). L'uroscanner avait conclu à une agénésie rénale droite avec masse kystique à contenu hyperdense, occupant la loge prostatique du coté droit et à développement intravésicale (Figure 3 et 4).

Correspondance :

DrAhmed CHOUKET - Service d'urologie et transplantation rénale, CHU Habib Bourguiba, Sfax, Tunisie -

$\mathrm{Tel}+21698564961$ - Email ahmedchouket@yahoo.fr 


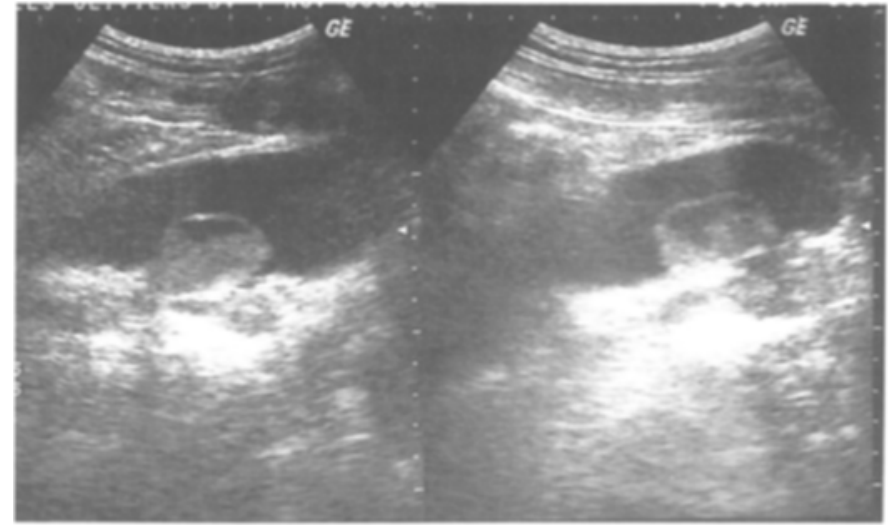

Figure 1 : Echographie pelvienne montrant une masse kystique de $3,5 \mathrm{~cm}$ de grand axe, à contenu hétérogène, rétrovésicale et faisant sailli dans la lumière vésicale.

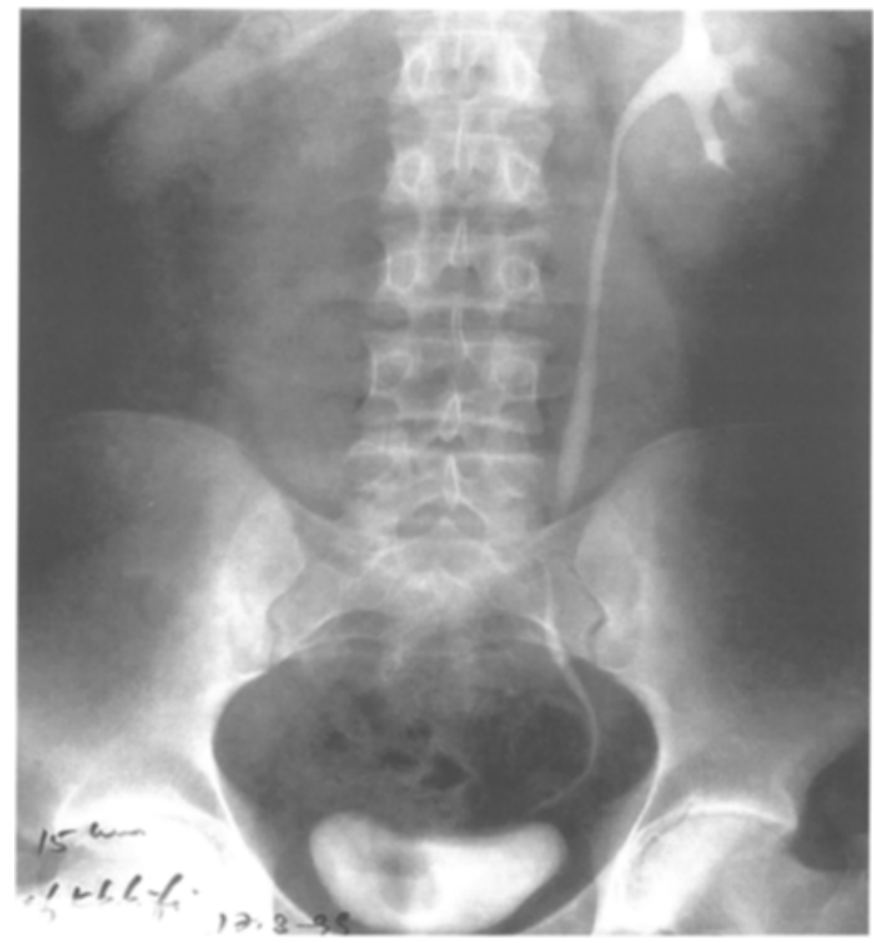

Figure 2 : UIV objectivant sur le cystogramme une image lacunaire droite du plancher vésical avec un rein droit muet.

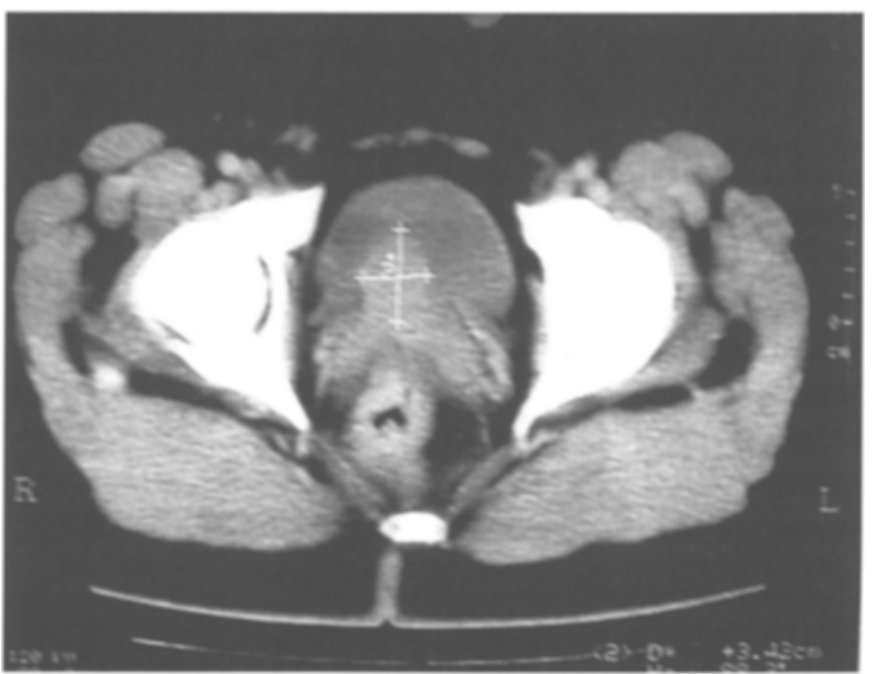

Figure 4 : Uroscanner ; masse kystique à contenu hyperdense, occupant la loge prostatique du coté droit et à développement intravésicale.

Figure 3 : Uroscanner ; agénésie rénale droite. 
Le patient a été opéré, ayant bénéficié d'une cystoscopie qui avait montré une formation kystique occupant l'hémitrigone droit de $4 \mathrm{~cm}$ arrivant jusqu'au col vésical et recouverte de muqueuse vésicale amincie. L'incision transversale de cette masse a fait sourdre du liquide épais brunâtre dont l'étude bactériologique n'a pas isolé de germe. L'injection du produit iodé à travers l'orifice du canal éjaculateur au niveau du veru-montanum a permis d'opacifier la vésicule séminale droite et la cavité kystique (Figure 5).

L'évolution était favorable cliniquement et radiologiquement avec disparition complète de la masse sur le scanner pratiqué après 3 mois de recul (Figure 6).

\section{Observation $\mathrm{N}^{\circ} 2$}

Il s'agissait d'un patient âgé de 16 ans, sans antécédents particuliers, hospitalisé pour masse kystique pelvienne de découverte fortuite à l'occasion d'une échographie abdominale faite pour un épisode de vomissement isolé. L'examen clinique était normal. L'uroscanner avait montré une agénésie rénale gauche avec présence d'une masse kystique à paroi propre, bien limitée, de siège rétrovésicale et faisant $5 \mathrm{~cm}$ de grand axe (Figures 7 et 8).

Le diagnostic d'un rein ectopique pelvien, hydronéphrotique, a été suspecté, et le patient a été exploré par laparotomie médiane sous-ombilicale. En per-opératoire la dissection était laborieuse et une kystectomie a été réalisée avec un drainage par redon aspiratif. L'examen anatomopathologique de la pièce opératoire a conclu à un kyste séreux de la vésicule séminale. Les suites opératoires étaient simples et le contrôle radiologique à 3 mois n'a pas montré d'anomalie pelvienne.

\section{Observation $\mathrm{N}^{\circ} 3$}

M. A. K., âgé de 34 ans, est admis au service d'urologie pour douleurs pelviennes fébriles associées à des troubles mictionnels à type de pollakiurie, dysurie et brûlures mictionnelles. Deux mois auparavant, il avait été hospitalisé et traité pour prostatite aiguë. A cette date, l'échographie pelvienne avait conclu à l'absence du rein droit et à un abcès prostatique devant la présence d'une image hypoéchogène, bien limitée siégeant dans la loge prostatique et dont le volume était de $250 \mathrm{~cm} 3$. Une ponction avait été pratiquée et l'étude cytobactériologique du liquide recueilli avait mis en évidence un Escherchia coli.

L'examen lors de cette deuxième admission retrouve, en dehors de la fièvre, un toucher rectal extrêmement douloureux et une douleur provoquée au niveau de l'hypogastre. Le reste de l'examen est sans particularité. L'échographie et l'uroscanner montrent des images évoquant une masse kystique rétrovésicale à paroi propre, refoulant la vessie en avant et à gauche, un rein gauche unique en hypertrophie compensatrice (Figures 9, 10 et 11).

L'abord extrapéritonéal est réalisé grâce à une incision iliaque, permettant d'individualiser une formation kystique dont la dissection est extrêmement difficile du fait de nombreuses adhérences à la vessie mais surtout à la prostate. A cette masse s'abouche l'uretère droit borgne. L'ensemble de ces structures est enlevé.

L'étude anatomopathologique met en évidence une vésicule séminale kystique, à contenu visqueux, brunâtre, à laquelle s'abouche l'uretère droit ectopique d'une part, et le canal déférent d'autre part. II s'agit d'un kyste de la vésicule séminale associé à une agénésie rénale droite et à un uretère ectopique ipsilatéral. Les suites ont été simples et le patient est actuellement asymptomatique.

\section{DISCUSSION}

Le KVS associé à une agénésie ou une dysplasie du rein homo latéral est rare [11]. Le nombre de cas publié jusqu'à maintenant n'excède pas les 200 [10].

L'origine congénitale d'un KVS est bien admise actuellement, surtout en présence d'une malformation associée de tractus uro-génital. La malformation la plus fréquente est l'agénésie rénale, suivi par l'abouchement ectopique de l'uretère ipsilatéral.

Les vésicules séminales et le bourgeon urétéral dérivent d'une même structure embryologique : le mésonéphros, et bien que leur croissance ne soit pas synchrone, il semble qu'une anomalie du développement du canal mésonéphrotique (à l'origine d'une malformation kystique de la vésicule séminale) interfère sur la croissance du bourgeon urétéral $[4,17]$. Survenant durant la gestation, l'obstruction urinaire avec un uretère ectopique peut être à l'origine d'une dysgénésie rénale. En effet, l'extrémité proximale du bourgeon urétéral ne rencontrant pas le blastème métanéphrogénique, elle favorise l'agénésie ou la dysgénésie rénale.

L'abouchement ectopique de l'uretère se fait dans le col de la vessie ou l'urètre prostatique $(54 \%)$, dans la vésicule séminale elle-même $(28 \%)$, ou dans le canal éjaculateur $(10 \%)[13,17]$. L'uretère est parfois absent et lorsqu'il est présent, il est borgne comme le cas de notre troisième observation. D'autres associations ont été décrites telles que un spina bifida, une duplicité urétérale, une anorchidie unilatérale ou une dysplasie rénale controlatérale $[15,16,18]$. L'hémivertèbre est une autre association rare au KVS, avec malformation rénale ipsilatérale [11]. 

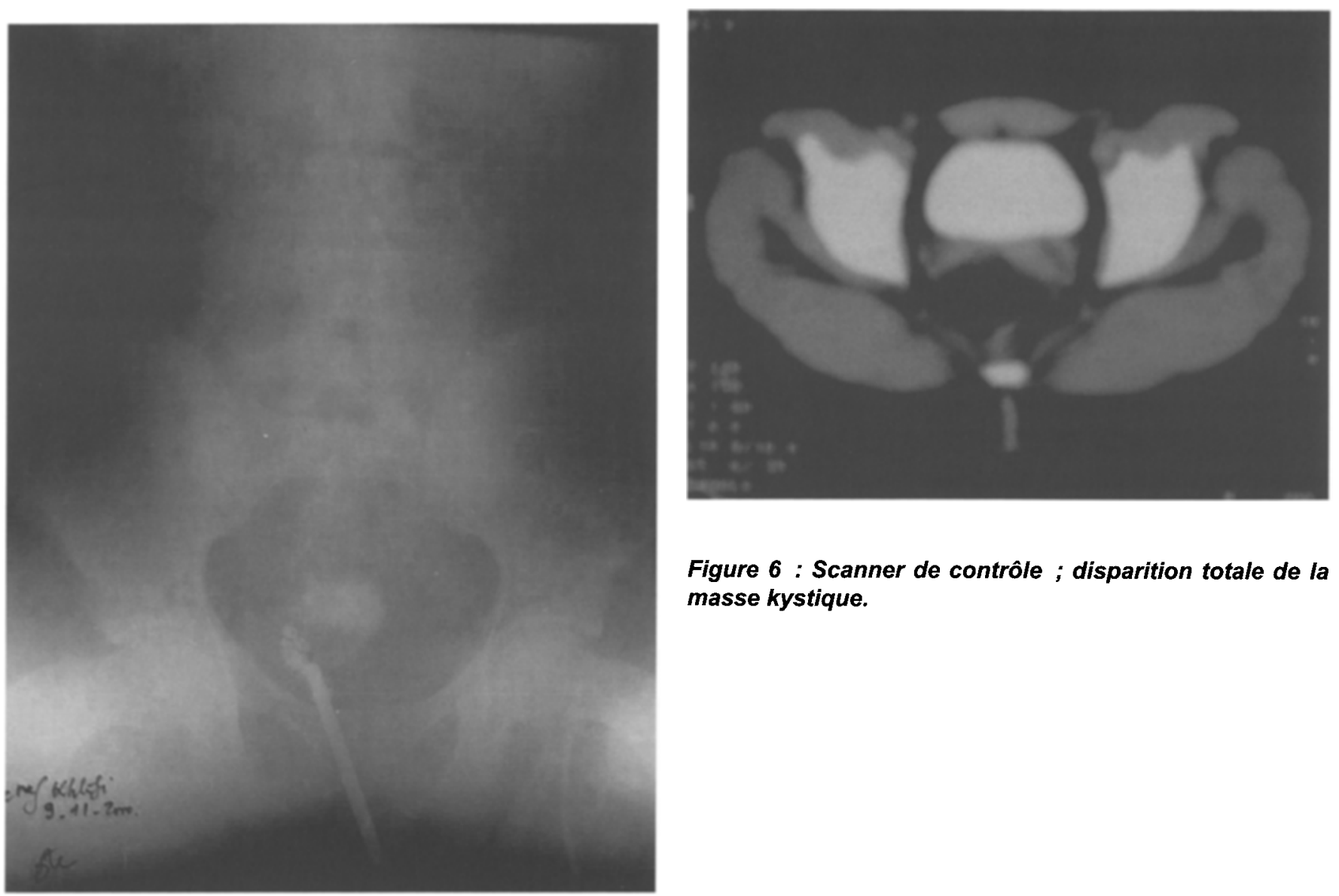

Figure 6 : Scanner de contrôle ; disparition totale de la masse kystique.

Figure 5 : Vésiculo-déférentographie : visualisation de la vésicule séminale droite avec opacification de la cavité kystique.

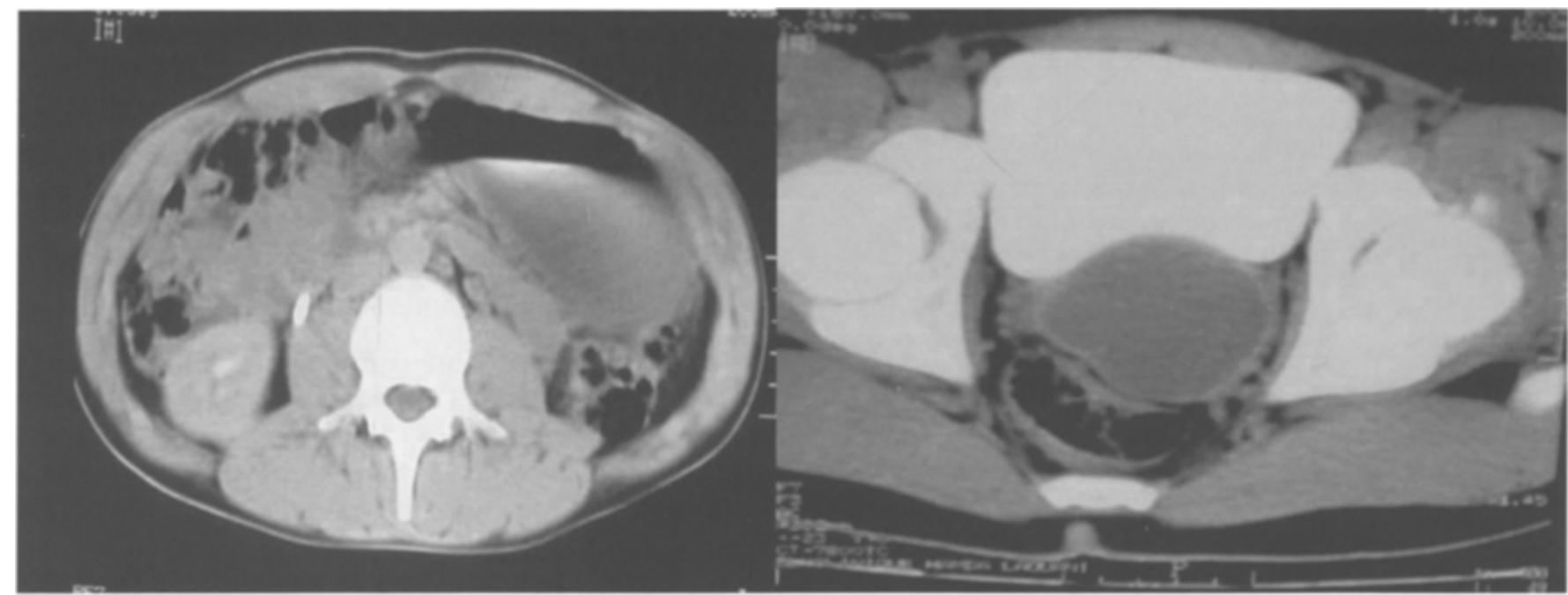

Figures 7 et 8 :Uroscanner; agénésie rénale gauche avec présence d'une masse kystique de $5 \mathrm{~cm}$ de grand axe, à paroi propre, bien limitée, de siège rétrovésicale. 


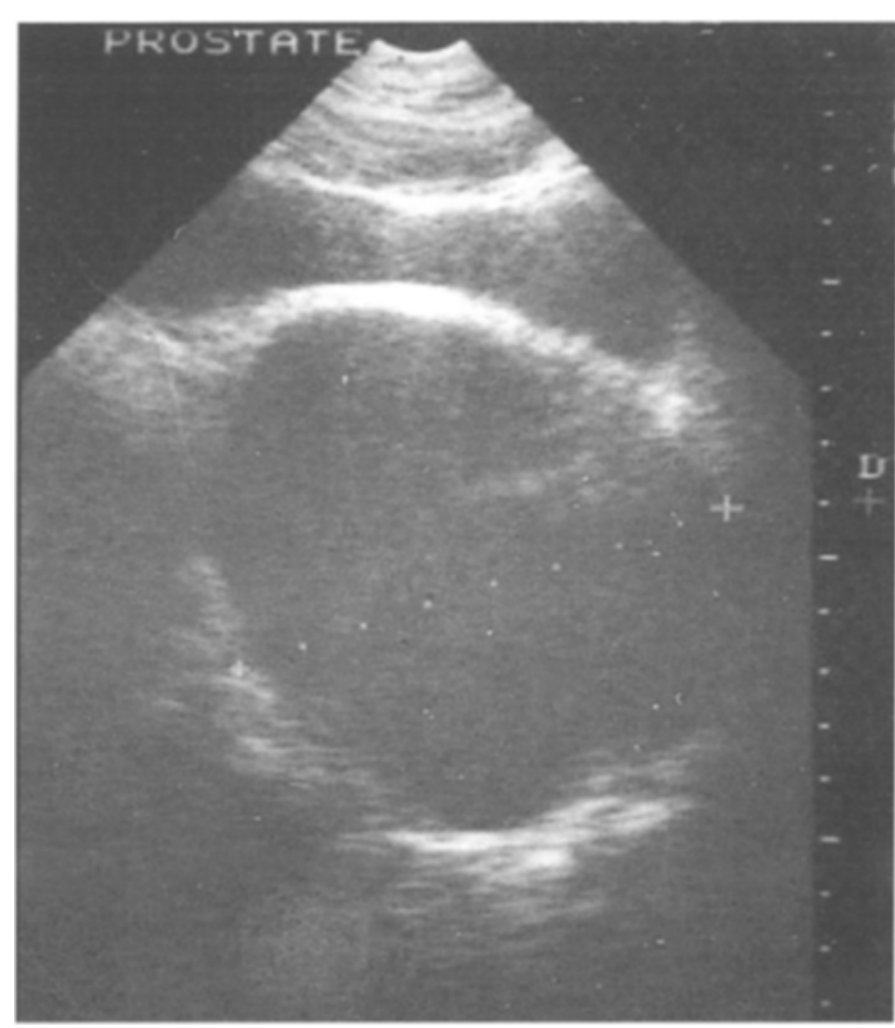

Figure 9 : Echographie pelvienne montrant une volumineuse masse kystique rétrovésicale.

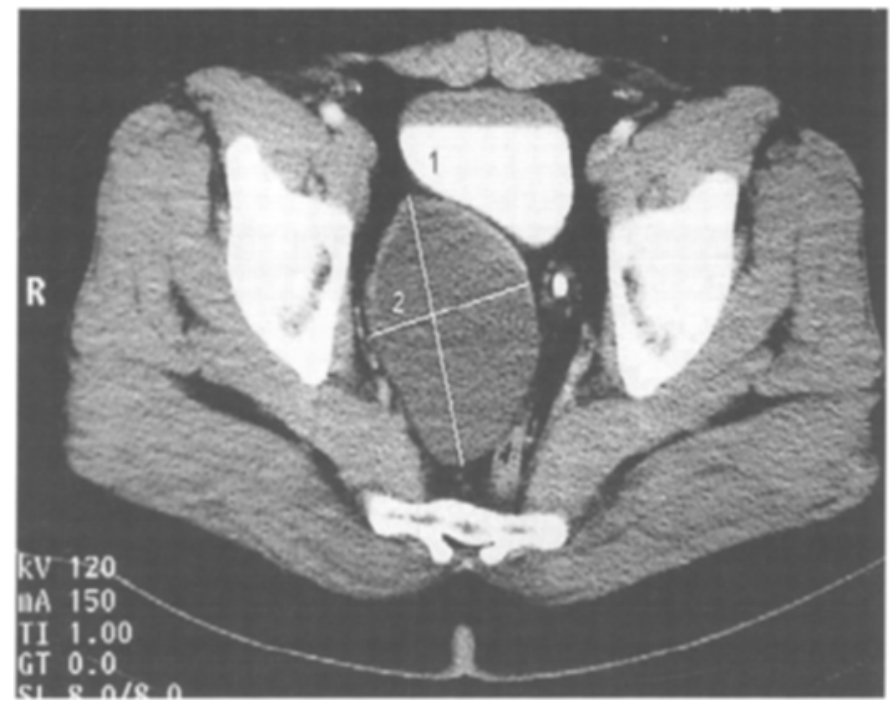

Figure 10 : Uroscanner : rein gauche unique, en hypertrophie compensatrice.

L'obstruction acquise du canal éjaculateur peut également être responsable du développement d'un KVS, secondaire à la distension de la glande par l'accumulation des sécrétions. Cette obstruction peut être secondaire à une infection génito-urinaire, une résection de prostate ou un calcul du canal éjaculateur $[8,10]$.

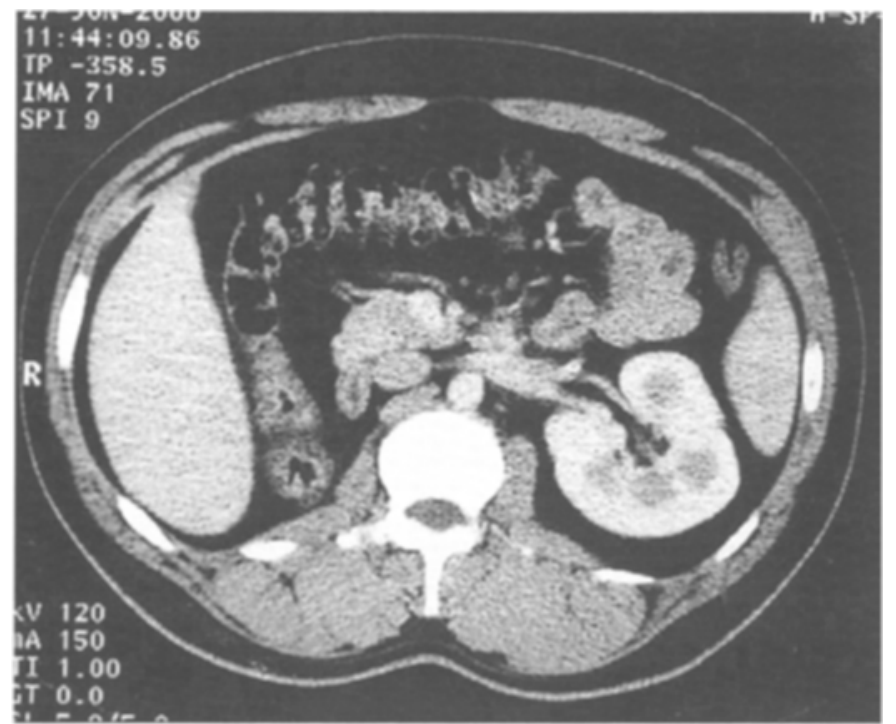

Figure 11 : Uroscanner ; masse kystique rétrovésicale à paroi propre, refoulant la vessie en avant et à gauche.

L'âge de découverte peut s'étaler tout au long de la vie, mais les lésions s'expriment principalement après la puberté chez l'adulte jeune.

Le diagnostic de cette anomalie est le plus souvent fortuit, fait à l'occasion d'une échographie ou d'une urographie intraveineuse pratiquée pour tout autre cause et montrant une hypoplasie ou agénésie rénale. Plus rarement, elle est responsable d'un syndrome irritatif du bas appareil urinaire avec parfois dysurie ou rétention vésicale, ou une douleur périnéale lors de l'éjaculation ou de la défécation, ou bien une infection urinaire, une épididymite ou une prostatite $[5,6,8,14,16,17]$. Ces infections sont le plus souvent récidivantes [8]. Dans notre troisième observation, il s'agit d'un tableau de prostatite aiguë avec fièvre et troubles mictionnels. Fatih et al. ont décrit un cas révélé par une constipation secondaire à une obstruction rectale chronique [6]. Le KVS peut se manifester également par une hémospermie ou une infertilité [10].

Le toucher rectal est un examen clé dans le diagnostic de cette pathologie. II permet de palper le kyste sous forme d'une masse rénitente qui occupe l'hémi-loge prostatique ipsilatérale. L'échographie endorectale est d'un grand apport diagnostique, elle trouve une image anéchogène à renforcement postérieur [16]. En cas de kyste compliqué d'hémorragie ou d'infection, son aspect devient hypoéchogène ou hétérogène avec présence d'échos internes comme dans le cas de notre troisième observation. L'échographie trans-rectale permet également de guider un geste de drainage du kyste qui peut être à visé thérapeutique ou cytobactériologique en cas d'infection [5]. 
La vésiculodéférentographie peut contribuer au diagnostic, en montrant soit une dilatation kystique de la vésicule séminale ou bien un kyste communiquant ou comprimant la vésicule. Dans notre premier cas, cet examen a montré le kyste communiquant avec vésicule séminale (Figure 5).

L'UIV montre souvent un refoulement du bas uretère homolatéral, l'empreinte du kyste sur la paroi vésicale ou une image lacunaire sur le cystogramme, ainsi que les malformations associés [12].

En cas de doute la TDM ou l'IRM rectifient le diagnostic et permettent de montrer les anomalies associées, notamment l'agénésie rénale $[4,9]$. Mais l'apport de I'IRM semble supérieur à la TDM en matière de pathologie kystique pelvienne; en effet, elle permet de rapporter le kyste à son origine et mieux étudier ses rapports avec les autres organes [9, 14].

Malgré le progrès de l'imagerie, un KVS peut poser encore le problème de diagnostic différentiel avec d'autres anomalies, telles que un kyste du canal éjaculateur ou des canaux des glandes prostatiques, un kyste du reliquat mullérien, une urétérocèle ectopique... $[4,8,9]$.

La majorité des équipes préfèrent que le traitement soit réservé aux formes symptomatiques ; les kystes de découverte fortuite ne doivent pas être traités [21]. Plusieurs options thérapeutiques ont été proposées pour traiter les formes symptomatiques, allant de la simple ponction-aspiration du kyste par voie transrectale ou transpérinéale, passant par la résection endoscopique, jusqu'à l'exérèse chirurgicale par voie ouverte ou laparoscopique $[2,3,10]$.

L'aspiration du kyste expose souvent à la récidive. La kystectomie ou la kysto-vésiculectomie est un geste qui reste souvent laborieux, surtout en cas d'antécédents infectieux. L'abord laparoscopique transpéritonéal est moins invasif, il permet une bonne exérèse du KVS [1$3,5,7]$.

Bien que quelques auteurs préfèrent la résection endoscopique de kyste, la majorité préfèrent un geste radical par voie transvésicale extrapéritoneale ou transpéritoneale extravésicale [21].

\section{CONCLUSION}

L'association d'un kyste de la vésicule séminale à une agénésie rénale et/ou une autre anomalie du tractus urinaire, telle l'abouchement ectopique de l'uretère, est une entité anatomo-clinique rare. Son diagnostic est devenu aisé grâce à l'imagerie faite pour des formes symptomatiques ou de découverte fortuite. Le traitement du KVS est très varié allant de la simple surveillance ou ponction jusqu'à l'exérèse chirurgicale laparoscopique.

\section{REFERENCES}

1. CARMACK A.J.K., SIDDIQ F.M., LEVEILLEE R.J. : Novel use of da vinci robotic surgical system : Removal of seminal vesicle cyst in previously dissected pelvis. Urology, 2006, 67: 199.

2. BASILLOTE J.B., SHANBERG A.M., WOO D. et al. Laparoscopic excision of a seminal vesicle cyst in a child. J. Urol., 2004, $17: 369-371$.

3. CHERULLO E.E., MERANEY A.M., BERNSTEIN L.H. et al.: Laparoscopic management of congenital seminal vesicle cysts associated with ipsilateral renal agenesis. J. Urol., 2002, $167: 1263-1267$

4. DAHMS S.E., HOHENFELLNER M., LIN J.F. et al Retrovesical mass in men : pitfalls of differential diagnosis. J. Urol., 1999, $161: 1244-1248$.

5. EVANGELOS N.L., BENJAMIN LEE., KRITON S.F. et al : Congenital seminal vesicle cyst and coexisting renal agenesis: laparoscopic approach. Urology, 2004, 63 : 584-586.

6. FATIHA., EDWARD D.K., FREDERICK A.K. et al. : Seminal vesicle cyst presenting as rectal obstruction. Urology, 2004, $63: 584-585$

7. IKARI O., CASTILHO L.N., LUCENAR. et al. : Laparoscopic excision of seminal vesicle cysts. J. Urol., 1999, 162 : 498499.

8. KARMOUNI T., EL FASSI M.J., EL KHADER K. et al. : Kyste de la vésicule séminale avec agénésie du rein homolatéral. Prog. Urol., 2002, 12 : 102-104.

9. LIVINGSTON L., LARSEN C.R. : Seminal vesicle cyst with ipsilateral renal agenesis. A.J.R., 2000, $175: 177-180$.

10. MANOUSAKAS T.H., KYRIAKOU G., SERAFETINIDES E. et al: Partial vesiculectomy in an infertile man with seminal vesicle cyst, ipsilateral renal agenesis, and cryptorchidism Urology, 2002, $59: 602$

11. MATSUKI M., MATSUO M., KAJI Y. et al. : Ectopic ureter draining into seminal vesicle cyst: unfulness of MRI. Radiat. Med., 1998, $16:$ 309-311.

12. MEGE J.L., SABATIER-LAVAL E., MURE P.Y. et al : Malformations des organes génitaux masculins issus du canal de Wolff (épididyme, déférent, vésicule séminale, canal éjaculateur). Prog. Urol., 1997, 7 : 262-269.

13. MOLLARD $P$ : Précis d'urologie de l'enfant. Paris, Edition Masson, $1984: 119-132$.

14. MURPHY J.O., POWER R.E., AKHTAR Met al: Magnetic resonance imaging in the diagnosis of seminal vesicle cysts and associated anomalies. J Urol., 2003, $170: 2386$.

15. PHILLIS T.M., TEAGUE J.L. : Seminal vesicle cyst in a child with a controlateral upper urinary tract abnormality. $\mathrm{Br}$. J. Urol. Int., 1999, $83:$ 359-360.

16. RAZI A., IMANI B. : Seminal vesicle cyst presenting with 
lower urinary tract symptoms and huge abdominal mass. J. Urol., 2000, $164: 1309-1310$.

17. SHEIH C.P., LI Y.W., LIAO Y.J., HUNG C.S. : Bilateral congenital cysts of the seminal vesicle with bilateral duplex kidneys. J. Urol., 1998, 160 : 184-185.

18. SHEIH C.P., LIAO Y.J., LI Y.W., YANG L.Y. : Seminal vesicle cyst associated with ipsilateral renal malformation and hemivertebra : report of 2cases. J. Urol., 1993,150:1214-1215.

19. ZAONTZ M.R., KASS E.J. : Ectopic ureter opening into seminal vesicle cyst associated with ipsilateral renal agenesis. Urology, 1987, $29: 523$.

20. ZINNER A : Ein fall von intravesikaler samenblasenzyste. Weien Med. Wschr., 1914, 64 : 604-610.

21. DUBOIS R., VALVALLE A.F., MURAT F. et al. : Malformations of male internal genital organs originating from the Wolff mesonephrotic canal. Prog. Urol., 2001,11: 733-740.

Manuscrit reçu : juillet 2006 ; accepté octobre 2006.

\section{ABSTRACT}

Seminal vesicle cyst associated with homolateral renal agenesis: report of three cases Ahmed CHOUKET, Mounir LEFI, Abdelkader BOUHLEL,
Abdelahad OUNAIES, Hamadi SAAD

The combination of seminal vesicle cyst and homolateral renal agenesis is a rare congenital anomaly. However, this anomaly is diagnosed more frequently due to progress in medical imaging.

The authors report three cases of seminal vesicle cyst associated with ipsilateral renal agenesis in two cases and ectopic ureter in one case. All cases presented nonspecific clinical symptoms.

The diagnosis was suspected on imaging (ultrasound and computed tomography). Treatment was surgical in two cases and endoscopic in one case.

The authors review the literature on the aetiology and pathogenesis of seminal vesicle cyst, its association with homolateral renal agenesis and diagnostic and therapeutic methods.

Keys Words: seminal vesicle, cyst, renal agenesis, aetiopathogenesis, cystectomy, endoscopic incision 